\title{
Preliminary Analysis of Contamination in Liver Preservation Solution and Prophylaxis for Post-transplant Infection
}

\author{
Zhao Jiqiang, 2, *, Zhao Jiquan ${ }^{2}$, Huo Feng ${ }^{3}$, Wang Shaping ${ }^{3}$, Zheng Yujian ${ }^{3}$ \\ ${ }^{1}$ Department of Organ Transplantation, The 3rd Affiliated Hospital, Guangzhou Medical University, Guangzhou, China \\ ${ }^{2}$ The Urinary Surgery, The Eighth Affiliated Hospital, SunYat-Sen University, Shenzhen, China \\ ${ }^{3}$ Department of Hepatobiliary Surgery, The liver Transplantation Center, Guangzhou General Hospital of Guangzhou Military of PLA, \\ Guangzhou, China
}

Email address:

mcjiqiangzhao@163.com (Zhao Jiqiang)

${ }^{*}$ Corresponding author

\section{To cite this article:}

Zhao Jiqiang, Zhao Jiquan, Huo Feng, Wang Shaping, Zheng Yujian. Preliminary Analysis of Contamination in Liver Preservation Solution and Prophylaxis for Post-transplant Infection. Science Discovery. Vol. 9, No. 4, 2021, pp. 171-177. doi: 10.11648/j.sd.20210904.17

Received: April 22, 2021; Accepted: June 4, 2021; Published: June 9, 2021

\begin{abstract}
Objective To discuss the preservation solution (PS) contamination and initial experience of liver transplantation from organ donation by citizens after death and initial experience. Methods The 78 liver transplant recipients were divided into positive group and negative group based on the finding of the culture of PS. The positive group received the sequential therapy of antibiotics with ertapenem and imipenem for one week, and the negative group stopped using imipenem. The situation of PS contamination and infection after liver transplantation and prognosis during the follow-up 3 months of the recipients were analyzed. Results PS culture positive rate was $41.03 \%$, and 33 strains of pathogens were isolated. The most common pathogenic bacteria were gram-negative bacilli (9 strains, 27.27\%) and coagulase-negative staphylococci ( 9 strains, 27.27\%). The infection rate after liver transplantation was $31.25 \%$ and $13.04 \%$, respectively, in positive group and negative group $\left(\chi^{2}=3.837, P=0.048\right)$. The most frequent infection sites were lower respiratory tract ( 5 cases, $31.25 \%$ ), abdominal cavity (5 cases, $31.25 \%$ ) and surgical incision (4 cases, 25.00\%). There was no significant difference in postoperative infection rate among patients with different CTP, MELD and surgical methods $(P>0.05)$. One case $(1.28 \%)$ was infected with the same pathogenic bacteria as PS contamination 3 weeks after liver transplantation, and died of multiple organ failure. There was no significantly difference in the acute rejection rate $(1,3.13 \%$ and $2,4.35 \%)$ and mortality $(2,6.25 \%$ and $5,10.87 \%)$ between the two groups $(P>0.05)$. Conclusion Contamination of the PS is frequent in liver transplantation, and it is the risk factor for postoperative infection of recipients. Early targeted antimicrobial treatment against pathogens cultured from PS play a positive role in reducing the contamination-associated infection rate after liver transplantation.
\end{abstract}

Keywords: Bacterial Contamination, Preservation Solution, Liver, Transplantation, Recipient Infection, Ertapenem

\section{肝移植保存液污染及其术后感染预防经验初步分析}

\author{
赵纪强 ${ }^{1,2 *}$, 赵济全 ${ }^{2}$, 霍枫 $^{3}$, 汪邵平 ${ }^{3}$, 郑于剑 ${ }^{3}$ \\ 1 广州医科大学附属第三医院器官移植科, 广州, 中国 \\ ${ }^{2}$ 中山大学附属第八医院泌尿外科, 深圳, 中国 \\ ${ }^{3}$ 广州军区广州总医院肝胆外科肝移植中心, 广州, 中国
}

邮箱

mcjiqiangzhao@163.com（赵济全） 


\begin{abstract}
摘要：目的：探讨公民逝世后器官捐献肝脏保存液（preservation solution, PS）污染及预防肝移植术后感染初步经验。 方法: 根据捐献肝脏PS培养结果将78例公民逝世后器官捐献肝移植受者分为阳性组和阴性组。阳性组围手术期序贯应 用厄他培南和亚胺培南, 疗程为一周; 阴性组停用亚胺培南。对保存液污染病原菌分布、术后感染和预后情况进行统 计分析。结果：PS培养阳性率 $41.03 \%$, 分离出 33 株病原菌, 最常见的病原菌是革兰氏阴性杆菌 $(9$ 株, $27.27 \%)$ 和凝固 酶阴性葡萄糖球菌 ( 9 株, $27.27 \%$ )。阳性组和阴性组肝移植受者术后感染率分别为 $31.25 \%$ 和 $13.04 \%\left(\chi^{2}=3.837, P=0.048\right)$ 。 感染例次最多的部位是下呼吸道（5例， $31.25 \%$ ）和腹腔（5例，31.25\%），其次是手术切口（4例， $25.00 \%$ ）。不同 肝功能Child-Turcotte-Pugh（CTP）、终末期肝病模型（model of end stage liver disease, MELD）评分以及手术方式患者 术后感染率差异无统计学意义。1例受者术后3周发生与PS污染相同的病原菌感染, 并因多脏器功能衰竭而死亡。在术 后随访3个月内，阳性组和阴性组患者急性排斥反应率和死亡率分别为 $3.13 \% （ 1$ 例）、4.35\%（2例）和6.25\%（2例）、 $10.87 \%$ （5例）, 差异均无统计学意义（ $P>0.05 ）$ 。结论: 公民逝世后捐献肝脏PS经常受到污染, PS污染是受者术后 感染的危险因素。早期针对性抗菌素治疗对降低公民逝世后捐献肝脏PS污染相关的感染有着积极作用。
\end{abstract}

关键词: 细菌污染, 器官保存液, 肝移植, 受者感染, 厄他培南

\section{1. 引言}

移植后感染是移植患者医院内发病和死亡的主要原 因，尤其是术后第一个月 $[1,2]$ 。器官保存液(preservation solution, PS)污染是术后感染的潜在来源。PS不但可以保 持污染菌存活, 而且有利于其生长, 从而提供了直接感染 器官受者的传播途径[3]。一项系统性综述和meta分析显示, 总PS培养阳性率为37\%（95\%CI:27\% 49\%）, PS培养致病 菌阳性的受者发生 PS 相关的感染为 $10 \%$ （95\%CI:7\% 15\%），培养阳性PS相关的感染增加移植受 者术后早期死亡率 $[4,5]$ 。为研究移植物PS污染并提高移植 术后感染相关并发症的早期诊断和管理, 许多器官移植中 心已常规进行移植前器官PS培养。然而, 目前还没有广为 接受的指南, 用于PS评估或预防性抗生素应用[6]。

本研究拟对肝移植供受者临床资料进行回顾性分析, 研究公民逝世后捐献肝脏器官保存液的污染发生率、病原 微生物类型和术后感染发生情况, 总结公民逝世后捐献器 官保存液污染和抗生素预防术后感染的初步经验, 从而为 提高肝移植临床疗效工作提供参考。

\section{2. 资料与方法}

\section{1. 资料来源}

选择广州市某三级甲等医院2016年3月至2017年11月 78例中国公民逝世后器官捐献肝移植患者为研究对象。多 器官联合移植、再次肝移植和跨血型肝移植患者不纳入本 研究。采用回顾性调查的方法, 收集肝移植供者和受者一 般资料、器官获取和肝移植手术信息、肝移植术后免疫抑 制剂方案、急性排斥反应、术后感染、病原学培养结果等 数据进行统计学分析。所有器官捐献者家属均签署知情同 意书, 符合医学伦理学规定。按照中国心脏死亡器官捐献 分类标准, 采取相应的捐献流程[7]。

\section{2. 快速规范化器官获取}

所有器官捐献手术均在手术室实施, 采用经腹主动脉 和门静脉联合灌注和腹部肝肾联合切取方式获取供肝,患
者腹主动脉插管灌注高渗枸橡酸盐腺嘌呤溶液 (Hypertonic Citrate-Adenine solution, HC-A液) 3000ml+威斯康星大学PS (University of Wisconsin solution, UW液) $1000 \mathrm{ml}$, 灌注高度 $100 \mathrm{~cm}$ 。肠系膜上静脉插管, 灌注 HC-A液 $3000 \mathrm{ml}+\mathrm{UW}$ 液 $500 \mathrm{ml}$, 灌注温度 $0 \sim 4^{\circ} \mathrm{C}$ 。供肝获取后应用器官保存袋逐层密 封后 $0 \sim 4^{\circ} \mathrm{C}$ 保存。供肝在手术室完成修整后留取供肝保存液 $100 \mathrm{ml}$ 进行需氧菌、厌氧菌和真菌培养。保存液有任何种类 细菌生长时, 则保存液培养阳性, 不考虑细菌种类。

\section{3. 手术方式及免疫抑制剂方案}

肝移植采取非转流经典原位肝移植或背驮式肝移植 术。受者术中及术后 $4 \mathrm{~d}$ 应用巴利昔单抗 ( Simulect ${ }^{\circledR}$, Novartis Pharma Schweiz AG) 20mg/d进行免疫诱导。无肝 期静脉应用甲基强的松龙 $(5-10 \mathrm{mg} / \mathrm{kg})$ 。术后第 1 天起, 甲基强的松龙从 $5 \mathrm{mg} / \mathrm{kg}$.day递减至 $0.3 \mathrm{mg} / \mathrm{kg}$.day，术后第 8 天停用。术后第一天开始口服麦考酚钠肠溶片 (540mg,q12h), 术后第四天开始口服他克莫司胶囊, 并 根据患者情况进行相应调整[8]。

\section{4. 围手术期抗生素预防及分组}

患者麻醉成功后开始静脉滴注厄他培南 $(1 \mathrm{~g}$, 输注时 间为 1 小时)。肝移植术后常规转入重症监护病房 (intensive care unit, ICU），病情稳定后转入肝移植病房进行专科治 疗。术后当日转换应用亚胺培南-西司他丁钠 $(1 \mathrm{~g}$, 静脉 滴注, q8h), 伏立康唑 (200mg, 静脉滴注, q12h)。 术后第三天根据PS培养结果将肝移植受者分为培养阳性 组和培养阴性组。阳性组维持原抗菌素方案, 疗程为一周。 阴性组停用亚胺培南。伏立康唑静脉滴注一周后改为口 服, 培养阴性组, 疗程2周; 培养阳性组, 疗程4周。

\section{5. 肝移植受者围手术期管理}

术后第一周每日检查肝功能、肾功能、电解质、血 常规、降钙素原、尿常规等指标, 腹腔引流液、尿液、 呼吸道分泌物细菌培养, 移植肝及各吻合管道彩超检 查、床边胸部X线摄片。当患者出现 2 项或以上炎症反应 综合征症状或体佂时, 则立即采集血液、尿液、痰等标 本进行细菌培养, 并增加培养次数。炎症反应综合征包 
括: 发热 $\left(>38^{\circ} \mathrm{C}\right)$ 或低体温 $\left(<36^{\circ} \mathrm{C}\right.$; 呼吸急促 $(>$ 20 次/分）或过度通气, $\mathrm{PaCO}_{2}<32.3 \mathrm{mmHg}$; 心动过速 ( $>90$ 次/分); 白细胞 $>12 \times 10^{9} / \mathrm{L}$ 或 $<4 \times 10^{9} / \mathrm{L}$, 或未成 熟白细胞 $>10 \%$ 。肝移植术后感染诊断参照 2001 年中华 人民共和国卫生部发布的《医院感染诊断标准》 [9], 根 据患者临床症状、体征、实验室及影像学结果确定。术 后患者一旦发生感染则进行相应治疗, 并依据细菌学培 养和药物敏感结果调整抗生素方案。依据移植肝穿刺活 检诊断急性排斥反应。

保存液细菌培养结果与供者和肝移植受者细菌培养 结果进行对比。病原菌匹配的标准是依据细菌种类及其相 应的药敏谱[10]。

\section{6. 统计方法}

数据使用SPSS 22.0软件进行数据统计分析。年龄分析 应用均数, 最大值和最小值表示, 计数资料以例数或百分 比表示。使用描述性统计和 $x^{2}$ 检验进行统计分析, $P<0.05$ 为差异具有统计学意义。

\section{3. 结果}

\section{1. 供者和受者基本资料}

\subsection{1. 供者资料}

78 例公民逝世后器官捐献供肝均由器官获取组织 （organ procurement organization, OPO）提供。其中阳性 组男性29例, 女性3例; 阴性组男性41例, 女性5例。阳性 组供者年龄16 61岁, 平均年龄39岁; 阴性组供者年龄 14 61岁, 平均38岁。根据中国心脏死亡器官捐献分类标 准, 属于中国一类, 即脑死亡器官捐献 (donation after brain death, DBD）供者47例; 中国二类, 即心脏死亡器官捐献 (donation after cardiac death, DCD) 5例; 其余26例为中 国三类, 即脑-心双死亡器官捐献 (donation after brain death awaiting cardiac death, DBCD）供者（表1）。供者死亡原 因包括严重脑外伤66例, 脑血管意外 10例和脑部神经系统 疾病2例（表2）。器官获取前阳性组供者ICU住院时间分 别为2 7天, 平均 4 天; 阴性组供者ICU住院时间为2 5天, 平均 3 天 $\left(x^{2}=29.938, P=0.000\right)$ 。

表1 纳入研究的公民逝世后器官捐献供者捐献类型分布。

\begin{tabular}{|c|c|c|c|c|c|c|c|c|}
\hline \multirow{2}{*}{ 分组 } & \multicolumn{2}{|l|}{ DBD } & \multicolumn{2}{|l|}{ DCD } & \multicolumn{2}{|c|}{ DBCD } & \multicolumn{2}{|l|}{ 合计 } \\
\hline & 例数 & 构成比 $(\%)$ & 例数 & 构成比（\%) & 例数 & 构成比 $(\%)$ & 例数 & 构成比 $(\%)$ \\
\hline 阳性组 & 23 & 48.94 & 0 & 0.00 & 9 & 34.62 & 32 & 41.03 \\
\hline 阴性组 & 24 & 51.06 & 5 & 100.00 & 17 & 65.38 & 46 & 58.97 \\
\hline 合计 & 47 & 100.00 & 5 & 100.00 & 26 & 100.00 & 78 & 100.00 \\
\hline
\end{tabular}

DBD:脑死亡器官捐献; DCD:心死亡器官捐献; DBCD:脑心双死亡器官捐献

表2 纳入研究的公民逝世后器官捐献供者死亡原因分布。

\begin{tabular}{lllllllll}
\hline \multirow{2}{*}{ 分组 } & \multicolumn{2}{l}{ 严重脑外伤 } & \multicolumn{2}{l}{ 脑血管意外 } & \multicolumn{2}{l}{ 脑部神经系统疾病 } & \multicolumn{2}{c}{ 合计 } \\
\cline { 2 - 9 } & 例数 & 构成比 $(\%)$ & 例数 & 构成比 $(\boldsymbol{\%})$ & 例数 & 构成比 $(\%)$ & 例数 & 构成比 $(\%)$ \\
\hline 阳性组 & 28 & 42.42 & 3 & 30.00 & 1 & 50.00 & 32 & 41.03 \\
阴性组 & 38 & 57.58 & 7 & 70.00 & 1 & 50.00 & 46 & 58.97 \\
合计 & 66 & 100.00 & 10 & 100.00 & 2 & 100.00 & 78 & 100.00 \\
\hline
\end{tabular}

\subsection{2. 受者资料}

接受公民逝世后器官捐献肝移植受者78例, 所有受者 均签署由医院伦理委员会批准的患者知情同意书, 符合医 学伦理学规定。阳性组男29例, 女3例, 年龄28 69岁, 平 均年龄49.72岁; 阴性组男41例, 女5例; 年龄31 72岁,
平均年龄 50.35 岁。受者基础肝脏疾病资料如表3所示, 受 者基础肝脏原发病包括: 原发性肝癌 (肝癌), 乙型病毒 性肝炎 (乙肝) 后肝硬化, 慢性重型肝炎, 胆管细胞癌 (肝 内胆管细胞癌）等。

表3 纳入研究的肝移植受者基础肝脏疾病构成比

\begin{tabular}{|c|c|c|c|c|c|c|c|c|c|c|c|c|}
\hline \multirow[b]{2}{*}{ 分组 } & \multicolumn{2}{|l|}{ 肝癌 } & \multicolumn{2}{|c|}{ 肝炎后肝硬化 } & \multicolumn{2}{|c|}{ 慢性重型肝炎 } & \multicolumn{2}{|c|}{ 胆管细胞癌 } & \multicolumn{2}{|l|}{ 其他 } & \multicolumn{2}{|l|}{ 合计 } \\
\hline & 例数 & $\begin{array}{c}\text { 构成比 } \\
(\%)\end{array}$ & 例数 & $\begin{array}{c}\text { 构成比 } \\
(\%)\end{array}$ & 例数 & $\begin{array}{c}\text { 构成比 } \\
(\%)\end{array}$ & 例数 & $\begin{array}{c}\text { 构成比 } \\
(\%)\end{array}$ & 例数 & $\begin{array}{c}\text { 构成比 } \\
(\%)\end{array}$ & 例数 & $\begin{array}{c}\text { 构成比 } \\
(\%)\end{array}$ \\
\hline 阳性组 & 20 & 40.82 & 6 & 37.50 & 4 & 57.14 & 1 & 50.00 & 1 & 25.00 & 32 & 41.03 \\
\hline 阴性组 & 29 & 59.18 & 10 & 62.50 & 3 & 42.86 & 1 & 50.00 & 3 & 75.00 & 46 & 58.97 \\
\hline 合计 & 49 & 100.00 & 16 & 100.00 & 7 & 100.00 & 2 & 100.00 & 4 & 100.00 & 78 & 100.00 \\
\hline
\end{tabular}

\section{2. 肝脏保存液细菌污染分析}

78例公民逝世后器官捐献者, 肝脏保存液病原菌培养 阳性32例, 阳性率 $41.03 \%$, 分离33株病原菌。其中革兰阳
性球菌 16 株占 $48.48 \%$, 革兰阴性杆菌9株占 $27.3 \%$, 酵母菌 5 株占 $15.15 \%$, 革兰阳性杆菌3株占 $9.09 \%$ 。病原菌分布情 况见表4。凝固酶阴性葡萄球菌、棒状杆菌、草绿色链球 菌群等系腐生菌群, 共 14 株, 占 $42.42 \%$; 而革兰氏阴性杆 
菌、金黄色葡萄糖球菌、草绿色链球菌之外的链球菌属、肠球菌和酵母菌等系致病菌，共19株，占57.58\%。

表4 公民逝世后捐献肝脏保存液培养病原菌构成比。

\begin{tabular}{lll}
\hline 病原菌 & 株数 $(\mathbf{n}=\mathbf{3 3})$ & 构成比 $(\mathbf{\%})$ \\
\hline 革兰阳性球菌 & 16 & 48.48 \\
金黄色葡萄球菌 & 1 & 3.03 \\
凝固酶阴性葡萄糖球菌 & 9 & 27.27 \\
肠球菌 & 4 & 12.12 \\
其他 & 2 & 6.06 \\
革兰阴性杆菌 & 9 & 27.27 \\
肠杆菌属 & 6 & 18.18 \\
恶臭假单胞菌 & 2 & 6.06 \\
鲍曼不动杆菌 & 1 & 3.03 \\
革兰阳性杆菌 & 3 & 9.09 \\
蜡状芽孢杆菌 & 2 & 6.06 \\
棒状杆菌 & 1 & 3.03 \\
酵母菌 & 5 & 15.15 \\
白假丝酵母 & 2 & 6.06 \\
热带假丝酵母 & 2 & 6.06 \\
光滑假丝酵母 & 1 & 3.03 \\
\hline
\end{tabular}

\section{3. 肝移植术后感染发生率及死亡率}

78例肝移植受者中术后3个月内，16例发生感染，感 染率为 $20.51 \%$ 。其中, 阳性组发生肝移植术后感染 10 例, 感染率为 $31.25 \%$, 阴性组发生肝移植术后感染 6 例, 感染 率为 $13.04 \%$, 阳性组感染率高于阴性组 $\left(x^{2}=3.837\right.$, $P=0.048 ） 。 78$ 例患者中，死亡7例，死亡率 $8.97 \%$ 。发生 感染的16例患者中，死亡3例，死亡率 $18.75 \%$ ，未发生感 染62例患者中死亡4例，死亡率 $6.45 \%$ ，死亡率比较差异无 统计学意义 $\left(x^{2}=2.355, P=0.148\right)$ 。
阳性组患者死亡2例, 死亡率 $6.25 \%$, 均死于术后感染; 阴性组患者死亡 5 例, 死亡率 $10.87 \%$, 其中死于术后感染 1 例, 术后腹腔出血2例, 病理证实的移植物超急性排斥反 应和围手术期多次心跳骤停致移植物功能衰竭各 1 例, 死 亡率比较差异无统计学意义 $\left(x^{2}=0.493, \mathrm{P}=0.391\right)$ 。

阳性组13例受者术后至少出现一次发热, 发生率 $40.63 \%$, 阴性组9例受者术后至少出现一次发热, 发生率 $19.57 \%$, 阳性组明显高于阴性组 $\left(x^{2}=4.133, \mathrm{P}=0.042\right)$ 。 术后二组患者 3 个月内发生经穿刺证实的急性排斥反应分 别为 1 例和 2 例, 排斥反应发生率之间的差异无统计学意义 (表5)。

表5 器官保存液污染对肝移植受者术后 3 个月感染率、急性排斥发生率、术后发热率和死亡率的影响。

\begin{tabular}{|c|c|c|c|c|c|c|c|c|}
\hline \multirow{2}{*}{ 分组 } & \multicolumn{2}{|c|}{ 感 染 } & \multicolumn{2}{|c|}{ 急性排斥反应 } & \multicolumn{2}{|c|}{ 术后发热 } & \multicolumn{2}{|c|}{ 死亡 } \\
\hline & $\mathrm{n}$ & $\%$ & n & $\%$ & $\mathrm{n}$ & $\%$ & $\mathrm{n}$ & $\%$ \\
\hline 培养液阳性 & 10 & 31.25 & 1 & 3.13 & 13 & 40.63 & 2 & 6.25 \\
\hline 培养液阴性 & 6 & 13.04 & 2 & 4.35 & 9 & 19.57 & 5 & 10.87 \\
\hline$P$ 值 & 0.04 & & & & 0.0 & & & \\
\hline
\end{tabular}

\section{4. 肝移植术后感染部位分布}

感染患者感染例次最多的部位是下呼吸道和腹腔，均为 5 例，均占 $31.25 \%$, 其次是手术切口， 4 例占 $25.00 \%$ 。不同 部位术后感染例次分布见表6。

表6 术后不同部位感染例次数构成比。

\begin{tabular}{|c|c|c|c|c|c|c|c|c|c|c|}
\hline \multirow{3}{*}{ 分组 } & \multicolumn{10}{|c|}{ 感染部位 } \\
\hline & \multicolumn{2}{|c|}{ 下呼吸道 } & \multicolumn{2}{|c|}{ 腹腔 } & \multicolumn{2}{|c|}{ 手术切口 } & \multicolumn{2}{|c|}{ 血液 } & \multicolumn{2}{|c|}{ 皮肤和软组织 } \\
\hline & $\mathbf{n}$ & $\%$ & $\mathbf{n}$ & $\%$ & $\mathrm{n}$ & $\%$ & $\mathbf{n}$ & $\%$ & $\mathbf{n}$ & $\%$ \\
\hline 阳性组 & 3 & 30.00 & 2 & 20.00 & 3 & 30.00 & 1 & 10.00 & 1 & 10.00 \\
\hline 阴性组 & 2 & 33.33 & 3 & 50.00 & 1 & 16.67 & 0 & 0.00 & 0 & 0.00 \\
\hline$P$ 值 & \multicolumn{2}{|c|}{0.331} & \multicolumn{2}{|c|}{0.669} & \multicolumn{2}{|c|}{0.185} & \multicolumn{2}{|c|}{0.571} & \multicolumn{2}{|c|}{0.571} \\
\hline
\end{tabular}

\section{5. 肝移植受者临床和手术指标分析}

为明确阳性组与阴性组之间肝移植受者因素差异, 对 受者临床和手术资料进行统计分析。结果显示，二组之间 各项指标差异无统计学意义（表7）。
1例严重脑外伤捐献者捐献前ICU住院6天，病原菌培 养阴性, 肝脏PS培养出鲍曼不动杆菌。该培养液阳性供肝 受者术后2周内肝功能恢复正常。术后第3周发生发热, 考 虑“移植物抗宿主病”, 血培养分离出鲍曼不动杆菌, 并于 术后第四周因重症肺部感染、多脏器功能衰竭死亡。1例 脑血管意外捐献者, 器官捐献前ICU住院6天, 痰培养分离 嗜麦芽寡养单胞菌, 肝脏PS培养分离头状葡萄糖球菌。该 
阳性受者术后4周因发热入院, 行ERCP (内镜下逆行胰胆 管造影术) 检查, 胆汁培养分离出鲍曼不动杆菌, 治疗后 症状缓解, 后胆管炎反复发作, 随访至术后 30 个月, 肝内
胆管已经出现广泛节段性狭窄性病变。其他PS培养阳性受 者术后恢复顺利, 随访至今, 人/移植物生存良好。

表7 阳性组与阴性组受者临床和手术指标之间比较。

\begin{tabular}{llll}
\hline 受者因素 & 阳性组 $\mathbf{n}=\mathbf{3 2}$ & 阴性组 $\mathbf{n}=\mathbf{4 6}$ & $\boldsymbol{P}$ \\
\hline CTP & $8.19 \pm 2.61$ & $8.41 \pm 3.09$ & 0.737 \\
MELD & $19.0 \pm 12.2$ & $18.7 \pm 10.5$ & 0.618 \\
手术方式 & & & 0.294 \\
经典式(n) & 21 & 34 & \\
背驮式(n) & 11 & 12 & 0.765 \\
无肝期 $(\mathrm{min})$ & $62.3 \pm 14.5$ & $63.4 \pm 17.5$ & 0.741 \\
冷缺血时间 $(\mathrm{min})$ & $301.6 \pm 82.8$ & $308.2 \pm 88.6$ & 0.435 \\
术中输红细胞量(u) & $10.0 \pm 7.2$ & $8.7 \pm 7.3$ & 0.287 \\
肝移植手术持续时间(h) & $7.4 \pm 1.8$ & $7.8 \pm 1.9$ & \\
\hline
\end{tabular}

CTP: Child-Turcotte-Pugh, MELD: Model for End-Stage Liver Disease

\section{6. 不同肝移植手术方式的术后感染情况}

78例肝移植患者中, 经典肝移植55例, 背驮式肝移植23例。不同手术方式患者的感染率比较差异无统计学意义 $\left(x^{2}=2.794, P=0.082\right)$ ，见表8。

表8 不同手术方式患者的术后感染率。

\begin{tabular}{llll}
\hline 手术方式 & 调查例数 & 感染例数 & 感染率 $(\%)$ \\
\hline 经典式肝移植术 & 55 & 14 & 25.45 \\
背驮式肝移植术 & 23 & 2 & 8.70 \\
\hline
\end{tabular}

\section{7. 不同CTP或MELD评分的患者术后感染情况}

78例肝移植患者中, 术前肝功能CTP评分, 其中A级 肝功能29例, B级肝功能22例, C级肝功能27例。其中B级 肝功能感染率最高, 为 $26.67 \%$, 但不同肝功能CTP评分患 者的感染率比较, 差异无统计学意义 $\left(x^{2}=5.044\right.$, $P=0.080 ） ， 见$ 表9。

术前MELD评分, 其中 $<20$ 分 51 例, $20 \sim 30$ 分 10 例, $30 \sim 40$ 分 15 例, $>40$ 分 2 例。MELD评分 $>40$ 分患者感染率 最高, 为 $50 \%,<20$ 分患者感染率最低, 为 $15.69 \%$ 。不同 MELD 评分患者的感染率比较差异无统计学意义 $\left(x^{2}=2.696, P=0.441\right)$ ，见表10。

表9 不同CTP肝功能分级患者的术后感染率。

\begin{tabular}{llll}
\hline CTP & 调查例数 & 感染例数 & 感染率 $(\%)$ \\
\hline A级 & 29 & 5 & 17.24 \\
B级 & 22 & 8 & 26.67 \\
C级 & 27 & 3 & 11.11 \\
\hline
\end{tabular}

表10 不同MELD评分患者术后感染率。

\begin{tabular}{llll}
\hline MELD & 调查例数 & 感染例数 & 感染率 $(\%)$ \\
\hline$<20$ 分 & 51 & 8 & 15.69 \\
$20 \sim 30$ 分 & 10 & 3 & 30.00 \\
$30 \sim 40$ 分 & 15 & 4 & 26.67 \\
$>40$ 分 & 2 & 1 & 50.00 \\
\hline
\end{tabular}

\section{4. 讨论}

本研究期间内, 78例公民逝世后捐献肝脏, PS培养阳 性率 $41.0 \%$, 与国外相关文献报道一致 [4]。肝移植受者发
生术后感染 16 例, 感染率为 $20.51 \%$, 低于国内相关文献报 道 $(54 \% \sim 83 \%)$ ～[11]。阳性组受者术后感染10例，感染率 $31.25 \%$, 而阴性组受者术后感染6例, 感染率 $13.04 \%$ 。可 见, 一旦PS污染, 肝移植受者术后发生感染风险显著增加。 肝移植术后 3 个月内总死亡率 $8.97 \%$, 发生感染的患者死亡 率为 $18.75 \%$, 无感染患者死亡率为 $6.45 \%$ 。发生感染患者 与无感染患者死亡率的差异无统计学意义, 我们推断与本 研究样本量少, 随访时间短有关。

目前, 国内对PS污染病原菌情况及其对移植后感染的 影响罕有报道。而污染的病原菌, 其可能来源于器官获取 时捐献者, 尤其是感染性捐献者, 以及腹部手术切口、消 化道管腔创口或肝脏修整等多个环节。污染的病原菌可通 过移植器官传播至受者而引起相应的术后感染等并发症。 由于培养时机、次数等因素影响, 器官获取前捐献者的血、 尿、呼吸道分泌物等细菌学培养, 并不能全面评估捐献器 官的感染状态。因此, 获取前器官捐献者各种标本与器官 PS病原学培养结果并不完全一致, 这与国外研究结果相似 $[12,13]$ 。因此, 器官PS病原菌培养对捐献器官评估、术后 抗生素选择、抗排斥药物使用等具有重要指导意义。

鉴于PS污染发生率很高, 但有理由推断, 缺乏感染传 播与早期使用有针对性抗生素有关。国外文献中, 很少有 报道, 通过污染的PS引起供者至受者的传播性感染 $[4,6,14]$ 。一旦发生经PS污染引起的感染传播案例, 其死 亡率非常高 $[5,15]$ 。此外, 现有的文献表明, 通过保存液 污染传播的疾病总是与已知的人类病原菌有关。当前研究 结果与其他研究相似, 78例公民逝世后捐献肝脏肝移植受 者, 1例发生与PS培养病原菌 (鲍曼不动杆菌) 相同病原 菌的术后感染, 于术后4周因重症肺部感染死亡。培养液 阳性肝移植受者术后虽然具有较高的术后感染率, 但死亡 
率没有增加。我们推断其原因: 一方面, 肝移植受者术后 无一例外均转入ICU监护治疗; 另一方面可能与术中和术 后序贯性应用长效、广谱抗菌药物有关。培养阳性受者具 有较高的发热率, 原因不明, 可能与循环中内毒素和其他 细菌产物有关 $[16,17]$ 。Chaim F H, et al[18]研究发现, PS 培养阳性受者更易发生急性排斥，原因是受者感染，降低 了免疫抑制剂强度而诱发排斥反应。但本研究中, 由于围 手术期序贯性使用碳青霉烯类广谱抗菌药物, 有效预防和 治疗PS污染及其他病原菌引起的受者感染, 可能降低了移 植肝急性排斥风险。

与其他研究结果相似, 本研究中PS培养最常见病原菌 是凝固酶阴性葡萄糖球菌和肠杆菌属。较高凝固酶阴性葡 萄糖球菌检出率, 提示细菌是外源性污染 (器官获取或者 供肝修整期间）而非内源性（来自肾或肝脏的细菌），提 示在器官获取、保存、运输和供肝修整等任何一个环节中 非无菌操作均是PS污染的潜在来源。更重要的是, 与其他 研究相似, PS中分离到更多的是致病菌, 包括肠杆菌、假 单胞菌和鲍曼不动杆菌等, 提示更应关注住院时间长, 尤 其是ICU存留时长的潜在器官捐献者的感染问题。一旦PS 出现致病菌生长时, 就应该考虑其受者的可能风险, 高度 重视PS致病菌污染可能引起的受者感染。

预防感染方面，相对于传统供肝肝移植，公民逝世后 供肝肝移植的感染更为严重, 常导致大出血等恶性并发 症。鉴于现阶段我院器官捐献供体来源多为严重脑外伤和 脑血管意外, 获取前入住ICU时间长, 存在气管插管、留 置尿管、深静脉置管或有创手术操作等医院内感染危险因 素, 甚至部分供者发生多重耐药菌感染并接受广谱抗生素 治疗 [19]。因此, 在前期研究基础上, 我中心在肝移植手 术期间采用厄他培南静脉滴注, 时间为 1 小时。厄他培南 具有覆盖需氧的革兰氏阳性和阴性以及庈氧菌的广谱抗 菌活性, 以及独特的更长的半衰期, 允许每日仅需使用一 次, 术中无需再次追加, 可保证手术期间有效的药物浓度, 从而便于手术管理[20]。术后当日转换应用亚胺培南-西司 他丁钠, 一方面具有与前者相似的抗菌活性, 另一方面具 有良好的性价比。更重要的是, 大量临床和实验研究显示, 厄他培南单独或与第二种碳青霉烯类抗生素在治疗器官 移植后复杂感染, 甚至耐碳青霉烯类细菌感染中显示了良 好的抗菌效果 [21-23]。供肝PS细菌药物敏感谱显示, 该序 贯性抗生素方案可完全覆盖器官PS污染病原菌, 并不需要 根据供肝PS培养结果再做针对性抗生素调整, 为术后治疗 可能来源于器官PS污染或术后受者感染争取了时间。结果 也显示, 培养阳性组受者死亡率、急性排斥发生率与阴性 组差异无统计学意义。因此, 我们推断, 序贯使用厄他培 南和亚胺培南方案能有效预防和治疗器官PS污染和术后 其他来源病原菌引起的受者感染。

本研究中, 5例公民逝世后捐献肝脏PS分离出酵母菌, 发生率 $6.4 \%$, 较其他研究结果稍高。通过移植物传播的念 珠菌症引起的真菌性动脉瘤炎与肾移植术后高发病率和 高死亡率具有相关性 $[24,25]$ 。然而, 在肝移植患者中鲜有 相关研究和报道。在本研究中, 5例PS培养阳性受者术后 恢复顺利, 未发生来自于PS污染的酵母菌感染, 可能与我 中心早期、足量和长疗程应用抗真菌药物有关。

\section{5. 结论}

综上所述, 公民逝世后捐献肝脏PS具有较高的细菌污 染率。随着我国公民逝世后器官捐献的开展, 捐献器官数 量和移植数量已经跃至世界第二位。但由于我国特有的器 官捐献模式、器官捐献者死亡原因、住院时间尤其是ICU 存留时间长, 潜在感染风险高。因此, 优化器官捐献和移 植前病原菌培养流程, 完善器官获取、保存和移植前器官 修整流程, 严格无菌操作, 有助于降低器官PS细菌污染风 险。围手术期序贯使用厄他培南和亚胺培南可有效预防和 治疗供肝PS污染和其他来源的病原菌引起的受者术后感 染。

然而, 由于研究样本少, 随访时间短, 且属于单中心 研究, PS污染对肝移植受者远期移植物功能和预后的影响 需要进一步研究。

\section{致谢}

基金项目: 深圳市卫生计生系统科研项目 (SZFZ2018044)。

\section{参考文献}

[1] Dorschner P, McElroy LM, Ison MG. Nosocomial infections within the first month of solid organ transplantation. Transpl Infect Dis, 2014; 16:171-87.

[2] Avkan-Oguz V, Ozkardesler S, Unek T, et al. Risk factors for early bacterial infections in liver transplantation. Transplant Proc, 2013; 45:993-7.

[3] Fishman JA. From the classic concepts to modern practice. Clin Microbiol Infect, 2014; 20:4-9.

[4] Oriol I, Sabé N, Tebé C, et al. Clinical impact of culture-positive preservation fluid on solid organ transplantation: A systematic review and meta-analysis. Transplantation Reviews, 2018; 32: 85-91.

[5] Janny S, Bert F, Dondero F, et al. Microbiological findings of culture-positive preservation fluid in liver transplantation. Transpl Infect Dis, 2011; 13: 9-14.

[6] Audet M, Piardi T, Panaro F, et al. Incidence and clinical significance of bacterial and fungal contamination of the preservation solution in liver transplantation. Transpl Infect Dis, 2011; 13:84-8.

[7] 中华医学会器官移植学分会. 中国心脏死亡器官捐献工作 指南 (第2 版) [J/CD]. 中华移植杂志: 电子版, 2012; 6(3):221-224.

[8] 中华医学会器官移植学分会. 中国肝移植免疫抑制治疗与 排斥反应诊疗规范( 2019 版) $[\mathrm{J} / \mathrm{CD}]$.中华移植杂志: 电 子版, 2019, 13(4) : 262-268.

[9] 中华人民共和国卫生部.医院感染诊断标准（试行）[J].中华 医药杂志, 2001;81(5): 314-320. 
[10] Oriol I, Lladó L, Vila M, et al. The Etiology, Incidence, and Impact of Preservation Fluid Contamination during Liver Transplantation. PLoS ONE, 2016; 11(8): e0160701.

[11] 蔵晓青, 钟林, 彭志海. 肝移植后感染的遗传易感性研究 [J]. 中华临床医师杂志(电子版), 2013;7(1): 238-240.

[12] Garcia-Zamora C, Segura J, Lopez-Lopez V, et al. Clinical Significance of Contamination of the Preservation Solution in Liver Transplantation. Transplant Proc, 2015; 47, 2322-23.

[13] Ruiz P, Gastaca M, Gonzalez J, et al. Incidence and Clinical Relevance of Bacterial Contamination in Preservation Solution for Liver Transplantation. Transplant Proc, 2009; 41, 2169-71.

[14] Sauget M, Verdy S, Slekovec C, et al. Bacterial contamination of organ graft preservation solution and infection after transplantation. Transpl Infect Dis, 2011; 13:331-4.

[15] Levesque E, Suet G, Merle JC, et al. Candida vascular complication in a liver transplant recipient due to yeast contamination of preservation solution. Transpl Infect Dis, 2014; 16(5):827-9.

[16] Nery JR, Weppler D, Ketchum P, et al: Donor infection and primary nonfunction in liver transplantation. Transplant Proc, 1997; 29: 481-83.

[17] Bud O, Golling M, Von Frankenberg M, et al. Intramucosal $\mathrm{pH}$ and serum endotoxin concentrations as early predictive parameters for primary nonfunction after experimental liver transplantation. Transplant Proc, 2000; 32: 2537-38.

[18] Chaim FH, Boin IF, Ataide EC,et al..Perfusion fluid contamination in relation to recipient survival and acute cellular rejection in orthotopic liver transplantation: retrospective analysis. Transplant Proc. 2011,43(4):1313-5.

[19] 赵纪强,霍枫,李鹏, 等. 中国心脏死亡器官捐献工作发展及 影响因素: 单中心经验 $[\mathrm{J} / \mathrm{CD}]$. 中华移植杂志: 电子版, 2017;11( 1) : 32-36.

[20] Shah PM, Isaacs RD. Ertapenem, the first of a new group of carbapenems. J. Antimicrob Chemother, 2003; 52:538-542.

[21] Goegele H, Berger N, Kafka R, et al. Course of transplant recipients treated with Ertapenem in the prophylaxis and treatment of infections: a first experience. Eur Surg, 2007; 39(3): 196-202.

[22] Oliva A,Scorzolini L, Cipolla A, et al. In vitro evaluation of different antimicrobial combinations against carbapenemase-producing Klebsiella pneumoniae: the activity of the double-carbapenem regimen is related to meropenem MIC value. J Antimicrob Chemother, 2017; 72: 1981-1984.

[23] Piedra-Carrasco N, Miguel L, Fàbrega A, et al. Effectiveness of a double-carbapenem regimen in a KPC-Producing Klebsiella pneumoniae infection in an immunocompromised patient. Microbial Drug Resistance, 2018; 24(2), 199-202.

[24] Botterel F, Foulet F, LegrandP,etal. Yeast contamination of kidney, liver and cardiac preservation solutions before graft: need for standardisation of microbial evaluation. J Hosp Infect, 2010; 76:52-5.

[25] Albano L, Bretagne S, Mamzer-Bruneel M-F, et al. Evidence that graft-site candidiasis after kidney transplantation is acquired during organ recovery: a multicenter study in France. Clin Infect Dis, 2009; 48: 194-202. 